\title{
Epidemiological Characteristics and Prediction of Esophageal Cancer Mortality in China from 1991 to 2012
}

\author{
Wen-Rui Tang¹, Jia-Ying Fang ${ }^{1}$, Ku-Sheng Wu ${ }^{1}$, Xiao-Jun Shi², Jia-Yi Luo ${ }^{1}$, \\ Kun Lin ${ }^{1 *}$
}

\begin{abstract}
Background: To analyze the mortality distribution of esophageal cancer in China from 1991 to 2012, to forecast the mortality in the future five years, and to provide evidence for prevention and treatment of esophageal cancer. Materials and Methods: Mortality data for esophageal cancer in China from 1991 to 2012 were used to describe its epidemiological characteristics, such as the change of the standardized mortality rate, urban-rural differences, sex and age differences. Trend-surface analysis was used to study the geographical distribution of the mortality. Curve estimation, time series, gray modeling, and joinpoint regression were used to predict the mortality for the next five years in the future. Results: In China, the incidence rate of esophageal cancer from 2007 and the mortality rate of esophageal cancer from 2008 increased yearly, with males at $8.72 / 10^{5}$ being higher than females, and the countryside at $\mathbf{1 5 . 5 / 1 0 ^ { 5 }}$ being higher than in the city. The mortality rate increased from age 45. Geographical analysis showed the mortality rate increased from southern to eastern China, and from northeast to central China. Conclusions: The incidence rate and the standardized mortality rate of esophageal cancer are rising. The regional disease control for esophageal cancer should be focused on eastern, central and northern regions China, and the key targets for prevention and treatment are rural men more than 45 years old. The mortality of esophageal cancer will rise in the next five years.
\end{abstract}

Keywords: Esophageal cancer - standardized mortality - geographic distribution - prediction - China

Asian Pac J Cancer Prev, 15 (16), 6929-6934

\section{Introduction}

The mortality rate of malignancy is gradually increasing. Worldwide, esophageal cancer is ranked eighth in incidence, sixth in mortality (Falk, 2009; Herszenyi et al., 2010; Chen et al., 2011). In the Chinese National Cancer Registry, the incidence of esophageal cancer is sixth and mortality fourth (Hao, 2012), with incidence and mortality rates significantly on the rise. This study analyzed monitoring data of cancer mortality rate in China from 1991-2012-in order to understand the dynamics of death, sex, age, urban and rural, and geographic distribution of esophageal cancer to determine the targets and regions of prevention and control, and to provide a scientific basis for prevention and treatment of esophageal cancer in China.

We used trend surface analysis to describe the geographical distribution of esophageal cancer mortality rate in China, not only to show the geographical distribution characteristics of the disease, but also to reflect the local disease diversity, which will provide important clues regarding the cause and effect of the relevant factors ( such as Sb, Sr, Se, Zn concentration in soil (Keshavarzi et al., 2012)). By using trend surface analysis charts (contour maps), we can provide a more intuitive understanding of the distribution of the disease in an area, so that people can glance the trends and geography distribution of the disease (Shi et al., 2014). Trend surface analysis is a new geographic epidemiological method that has been used in the study of epidemics.

\section{Materials and Methods}

\section{Data Source}

Data came from the nation-wide cancer mortality survey of the Chinese National Cancer Control Office for the period 1991-2012, Ministry of Public Health and the national sample survey from 2006 to 2012 (Chen, 2008; Zhao, 2008; Zhao, 2009; Zhao, 2010; Zhao, 2011; Hao, 2012). Data collection followed the rules and standards of the International Agency for Research on Cancer (IARC) and the International Association for Cancer Registration.

Evaluation of data quality was based on diagnosis reliability, data integrity and coding quality. By calculating the statistical magnitude, significance test of sample mean, goodness of fit test of national samples, regional samples and the overall have not statistically significant difference. A goodness of fit test of population age structure indicated 
that the overall sample had a better demographic representation. The gender composition of the population demonstrated that the age sex ratio was reasonable and could represent the national situation. The reliability of diagnosis was based on the distribution of the deceased highest diagnostic region and the highest death diagnosis and the integrity of death data was evaluated according to the mortality distribution of the survey counties (cities). The accuracy of death coding was used to determine the cause of coding quality.

\section{Statistical Analysis}

Standardized mortality of EC in the 1982 population was used to standardize mortality trends, to compare mortality changes in time, between people and geography.

\section{Methods}

On the basis of standardized mortality of different gender, the mean $(\mathrm{z})$ of standardized mortality, latitude (y) and longitude (x), from 40 countries in China, were used for binary polynomial regression to fit in a trend surface analysis, to describe the geographic distribution of esophageal cancer mortality for different gender. SAS9.1.3 software was used to complete trend surface analysis and contour maps.

This study aimed to estimate the trend of EC mortality in China from 1991 to 2012 and to predict the following 5-year trend. Epidemic trends of EC could be predicted by using curve estimation, time series modeling, gray modeling (GM) and joinpoint regression. The information could help to set up an early warning system for EC and provide public health agencies with a scientific basis to carry out disease prevention and control. Statistical analysis was performed by SPSS19.0, DPS 9.50 and Joinpoint 4.1.0.

Curve estimation were fitted to the data to quickly estimate regression statistics and produce related plots for 11 different models (linear, logarithmic, inverse, quadratic, cubic, power, compound, S-curve, logistic, growth and exponential models). In this investigation, $\mathrm{x}$ stands for the time (year) and y stands for standardized mortality rates (SMR).

Given an observed time series, the first step in analyzing a time series is to plot the given series on a graph taking time intervals $(t)$ along the $\mathrm{X}$-axis (as the independent variable) and the observed value $\left(Y_{t}\right)$ on the Y-axis (as the dependent variable). Such a graph will show various types of fluctuations and other point of interest. The autocorrelation function, partial autocorrelation function, mean squared error and mean absolute deviation were selected to be the forecasting accuracy measures (Imdadullah, Retrieved 11 June 2014).

When information was comparatively insufficient, GM $(1,1)$ was employed to predict the change of EC in China. GM $(1,1)$ can effectively describe the characteristics of some outputs using less information (at least four numbers). Numbers 1-22 were the time dependent variables, and the SMR was the independent variable and were imported to construct models in DPS software. The improved GM $(1,1)$ model group and dynamic equal dimensional number progress complement was adopted in order to obtain more accurate forecasts and to avoid the deficiencies of the one-direction gray model, which is easily affected by unstable information.

The joinpoint model was used to analyze EC mortality trends, find out the estimated tendency and get the annual percentage rate change and average annual percent change of EC in China.

\section{Results}

Esophageal cancer incidence and mortality trends from 2003 to 2012

The standardized incidence of esophageal cancer steadily rose from the 1990 s to 2012 , with esophageal cancer incidence being $22.14 / 10^{5}$ in 2011 , the standardized incidence rate $10.88 / 10^{5}$, and the incidence rate in rural areas being 2.7 times than that in the city (Hao, 2012). Esophageal cancer mortality ranked fourth in China as a whole, with standardized mortality rates steadily increasing an average $1.06 / 10^{5}$ per year. Esophageal cancer mortality was $16.77 / 10^{5}$ in 2012 , the standardized mortality was $7.75 / 10^{5}$, and the mortality rate in rural areas was 2.2 times than the cities (Hao, 2012).

\section{Differences of esophageal cancer mortality between urban and rural areas}

Since the 1990s, esophageal cancer mortality rose steadily and mortality rates were significantly higher in rural areas than in urban areas; esophageal cancer mortality had an average of $15.48 / 10^{5}$ higher in rural areas than in urban areas from 2003 to 2011 . Esophageal cancer mortality ranked $5^{\text {th }}$ in urban areas and ranked $2^{\text {th }}$ in rural areas. In 2011, the urban esophageal cancer standardized mortality was $4.65 / 10^{5}$, while in rural areas it was $18.64 / 10^{5}$ (Hao, 2012).

Gender and age-group characteristics of esophageal cancer mortality

Since the nineties esophageal cancer showed significant gender-dependent differences, with males having higher incidence than females, and mortality being $8.72 / 10^{5}$ higher on average in men than women from 2003 to 2011 . Esophageal cancer mortality ranked $4^{\text {th }}$ in menand $6^{\text {th }}$ in women. The esophageal cancer standardized mortality for men was $11.42 / 10^{5}$ in 2011 , while in women the mortality was $4.22 / 10^{5}$ (Zhao, 2008; Zhao, 2009; Zhao, 2010; Zhao, 2011; Hao, 2012).

Age-specific mortality rates of esophageal cancer remained a low level in the 0 to 44 years age group, and started to rise significantly in 45 year old group, reaching the highest in the 80 to 84 age group. In the 45 to 49 age group, the mortality rose to $25.40 / 10^{5}$ in rural areas, while it rose to $17.92 / 10^{5}$ in city in the 50 to 54 age group. Rural and urban mortality peaked at $229.08 / 10^{5}$ and $119.14 / 10^{5}$ in the 80 84 age group, respectively. Age-specific mortality changed significantly in rural areas, and the rapid increase in the rural areas was a fiveyear-old age group in advance than in the urban areas, peaking in the 80 to 84 age group. Mortality began to rise in males 40 to 44 years-old and was highest in the 80 to 84 age group. In women, mortality began to rise in 45 to 
49 years-old, a maximum in the 75 to 79 age group. In the rural areas, male mortality peaked in the 75 to 79 age group $\left(452.26 / 10^{5}\right)$, whereas in cities, mortality reached the highest in the 80 to 84 age group $\left(137.52 / 10^{5}\right)$. Rural female mortality was the highest in the 70 to 74 age group $\left(217.49 / 10^{5}\right)$, while in city it was the highest in the age group of 80 to $84\left(51.50 / 10^{5}\right)$ (Figures 1 and 2).

Geographical distribution of esophageal cancer mortality

According to esophageal cancer mortality rates for different genders in various observation regions, and their corresponding geographical locations, a binary polynomial regression equation was built for trend surface analysis. A meaningful $\left(F=7.33, p<0.001, R^{2}=0.5187\right)$

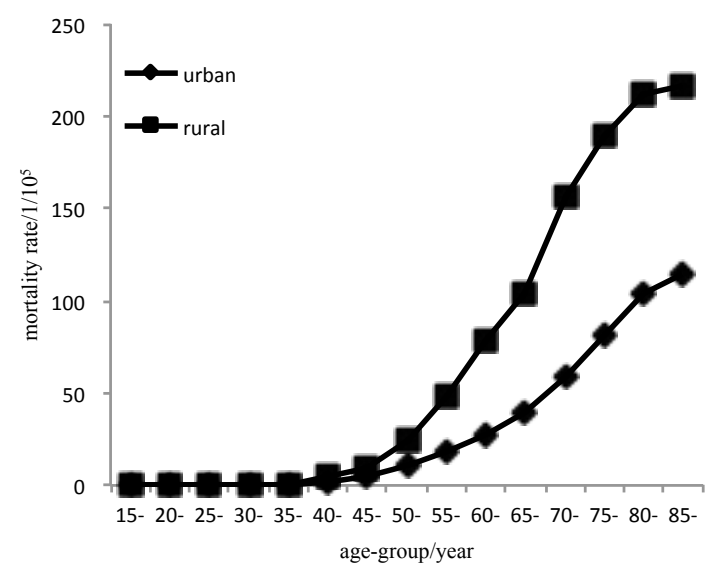

Figure 1. Change in Age-Specific Mortality of Esophageal Cancer between Urban and Rural Area sin China from 2003 to 2012

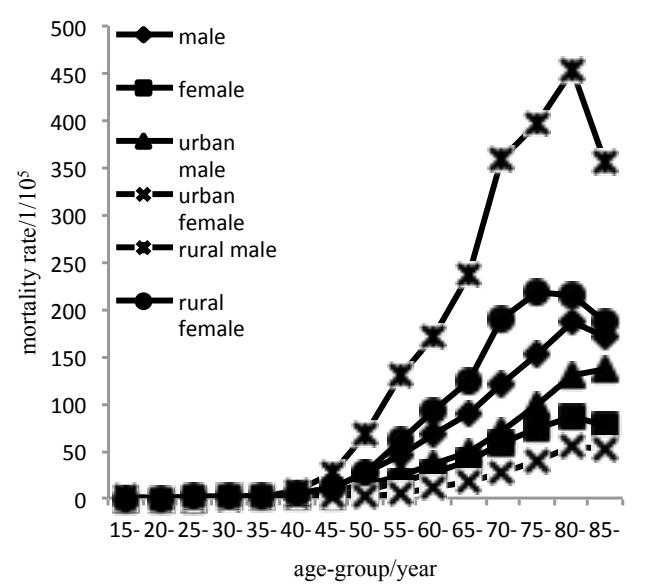

Figure 2. Change in Age-Specific Mortality of Esophageal Cancer among Urban and Rural Areas, and Different Sex in China from 2003 to 2012

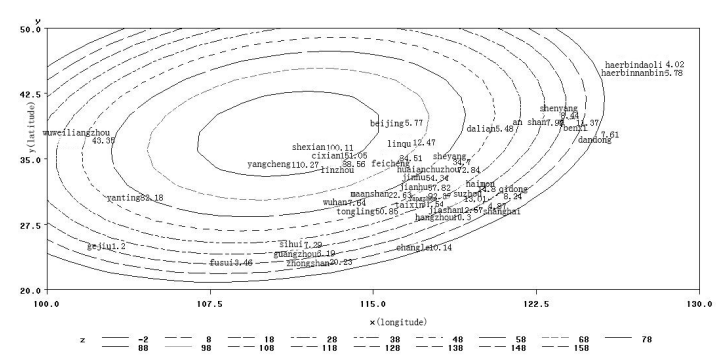

Figure 3. Geographical Distribution of the Standardized Mortality Rate of Male Esophageal Cancer in China second-order trend surface equation, for esophageal cancer mortality trends in men of the geographic distribution of esophageal cancer mortality, can be predicted using:

$z=-5031.5674+90.3963 * \mathrm{x}+5.0727 * \mathrm{y}-$ $0.4361 * \mathrm{x} * \mathrm{x}+0.1717 * \mathrm{x} * \mathrm{y}-0.3191 * \mathrm{y} * \mathrm{y}$.

Similarly, the following second-order equation for esophageal cancer mortality trends in women was also meaningful $\left(F=5.68, p=0.0007, R^{2}=0.4552\right)$ :

$z=-2168.2761+39.8040 * \mathrm{x}+0.0958 * \mathrm{y}-$ $0.2021 * \mathrm{x} * \mathrm{x}+0.1409 * \mathrm{x} * \mathrm{y}-0.2213 * \mathrm{y} * \mathrm{y}$.

Where $\mathrm{z}$ stands for the standardized mortality ratio of esophageal cancer for various cities/towns, $x$ stands for longitude of the cities/towns, and $y$ stands for latitude.

These two models illustrate that geographical environment factors might explain the variation $(51.87 \%$ and $45.52 \%$ ) of male and female esophageal cancer mortality rates, respectively, i.e. esophageal cancer mortality rates have a certain geographical distribution in different genders. Mortality rates increased gradually from a minimum in southern China (Fusui, Sihui) to elevated levels in eastern China. Gradual increases were also observed from northeast to the highest levels in the central region, revealing a semi-concentric geographical distribution. Minimum in the South China (Fusui, Sihui), Central China, high in North China (Ci County, Yangcheng County, SheXian, Linzhou); then the mortality rates also showed a gradual increase from eastern China to central China (Figures 3 and 4).

\section{Estimation and forecasting}

The sample of latest data is small, and is not credible to assume the derived distribution. Therefore, several predictive models with different statistical methods were built and the results were compared with each other in order to find a suitable trend for mortality of EC in future years. It was assumed that the society, economy, environment and lifestyle had no dynamic and highly variable characteristics. This made the previous EC mortality data, from 1991-2012, suitable for establishing a predictive model. The fitted error value and the $95 \%$ confidence interval $(\mathrm{CI})$ were selected to describe the forecasting accuracy. Four models were built to predict the mortality of EC.

Curve estimation: The power curve fitted the trend best $\left(R^{2}=0.778, F=70.048, p<0.001\right), y=(2.71 \mathrm{E}-197) *$ ( $\mathrm{x} * * 59.856)$, where $x$ stands for year, and $y$ stands for mortality rate (Tables 1 and 2 ).

Time series: According to the above mortality data, the built autoregressive integrated moving average (ARIMA) model was employed for prediction $\left(R^{2}=0.795, p=0.552\right)$

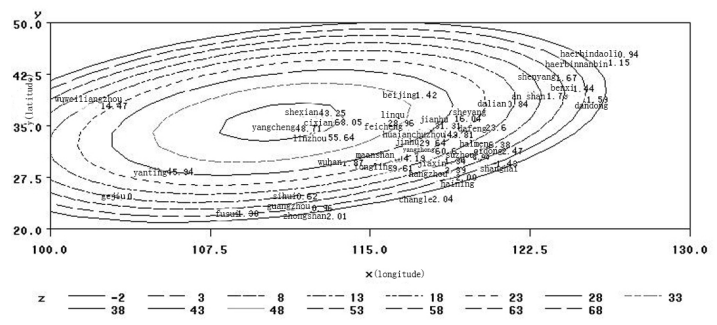

Figure 4. Geographical Distribution of the Standardized Mortality Rate of Female Esophageal Cancer in China 
Table 1. The Modeled Mortality for EC, by 4 Models, in China from 1991 to $2012\left(1 / 10^{5}\right)$

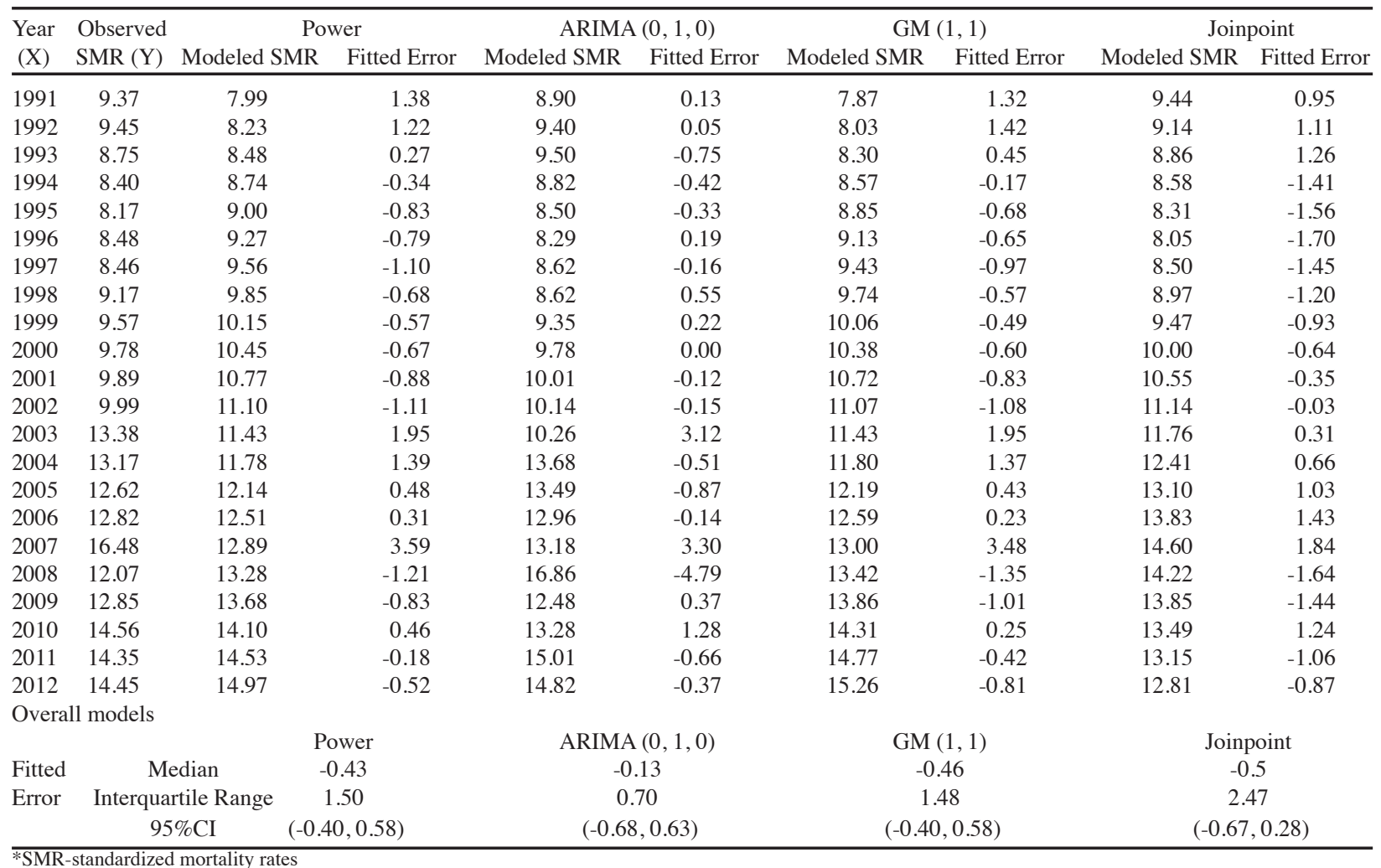

Table 2. Predicted Mortalities for EC, by 4 Models, in China During 2013-2017 (1/10 $)$

\begin{tabular}{|c|c|c|c|c|c|c|}
\hline \multirow[t]{2}{*}{ Year } & \multirow[t]{2}{*}{ Power } & \multirow{2}{*}{$\begin{array}{c}\text { ARIMA } \\
(0,1,0)\end{array}$} & \multirow{2}{*}{$\begin{array}{l}\text { GM } \\
(1,1)\end{array}$} & \multirow[t]{2}{*}{ Joinpoint } & \multicolumn{2}{|c|}{ Overall } \\
\hline & & & & & $\mathrm{X} \pm \mathrm{SD}$ & $95 \% \mathrm{CI}$ \\
\hline 2013 & 15.420 & 14.946 & 15.753 & 15.834 & $15.49 \pm 0.40$ & $(15.06,15.79)$ \\
\hline 2014 & 15.881 & 15.938 & 16.266 & 16.846 & $16.23 \pm 0.44$ & $(15.90,16.62)$ \\
\hline 2015 & 16.368 & 16.421 & 16.796 & 17.393 & $16.74 \pm 0.47$ & $(16.38,17.15)$ \\
\hline 2016 & 16.856 & 16.918 & 17.344 & 17.974 & $17.27 \pm 0.52$ & $(16.87,17.71)$ \\
\hline 2017 & 17.344 & 17.441 & 17.909 & 18.591 & $17.82 \pm 0.57$ & $(17.37,18.30)$ \\
\hline $\mathrm{X} \pm \mathrm{SD}$ & $16.37 \pm 0.76$ & $16.33 \pm 0.96$ & $16.81 \pm 0.85$ & $17.33 \pm 1.06$ & & \\
\hline $95 \% \mathrm{CI}$ & $(15.79,16.95)$ & $(15.54,17.04)$ & $(16.17,17.47)$ & $(16.46,18.12)$ & & \\
\hline
\end{tabular}

Table 3. APC a and $\mathrm{AAPC}^{\mathrm{b}}$ of age-standardized rates for $\mathrm{EC}$ in China from 1991-2012

\begin{tabular}{lcccc}
\hline Trends & APC $^{\mathrm{a}} / \mathrm{AAPC}{ }^{\mathrm{b}}$ & Urban & Rural & Overall \\
\hline Segment 1 & Years & $1991-1996$ & $1991-2001$ & $1991-1996$ \\
& APC $(95 \% \mathrm{CI})$ & $-9.5^{\mathrm{c}}(-17.2,-1.2)$ & $0.1(-0.9,1.1)$ & $-3.1(-8.3,2.4)$ \\
Segment 2 & Years & $1996-2001$ & $2001-2004$ & $1996-2007$ \\
& APC $(95 \% \mathrm{CI})$ & $14.8^{\mathrm{c}}(1.1,30.5)$ & $22.6^{\mathrm{c}}(2.2,46.9)$ & $5.6^{\mathrm{c}}(3.5,7.6)$ \\
Segment 3 & Years & $2001-2005$ & $2004-2012$ & $2007-2012$ \\
& APC $(95 \% \mathrm{CI})$ & $-22.8^{\mathrm{c}}(-37.6,-4.5)$ & $0.5(-0.9,1.9)$ & $-2.6(-7.8,3.0)$ \\
Segment 4 & Years & $2005-2012$ & $\mathrm{ND}^{\mathrm{d}}$ & $\mathrm{ND}^{\mathrm{d}}$ \\
& APC $(95 \% \mathrm{CI})$ & $21.0^{\mathrm{c}}(14.9,27.3)$ & $1991-2012$ & $\mathrm{ND}^{\mathrm{d}}$ \\
Full Range & Years & $1991-2012$ & $3.2^{\mathrm{c}}(0.7,5.7)$ & $1991-2012$ \\
& AAPC $(95 \% \mathrm{CI})$ & $2.4(-2.7,7.7)$ & & $1.5(-0.5,3.4)$ \\
\hline *APC & & &
\end{tabular}

*APC ${ }^{\mathrm{a}}$-annual percent change; AAPC ${ }^{\mathrm{b}}$-average annual percent change; ${ }^{\mathrm{c}}$-significantly different from zero at alpha $=0.05(p<0.05) ;{ }^{\mathrm{d} N D}$-no data

(Tables 1 and 2).

Gray model: According to the theory and principle of the gray model, $X(i)$ stands for the standardized mortality rate of EC, and $t$ stand for the year. The GM $(1,1)$ model was performed to forecast with fitting parameters: $a=-$ $0.0321, b=7.6064$. The equation is:

$X(t+1)=246.6245 \mathrm{e}+0.03206 \mathrm{t}-237.2545(C=0.4753$, $p=0.9048, Q \min =-4.1741)$ (Tables 1 and 2).

Joinpoint model: To fit the model, the dependent variable $y$ was the mortality rate, and independent variable $\mathrm{x}$ was the year, and joinpoint regression analysis was performed. The best fitting points were selected (Tables 1 and 2), and used to calculate the estimated annual percent change and average annual percent change in China during 1991-2012 (Table 3).

\section{Discussion}

We showed that the SMR of EC in China has been steadily rising and the trend is predicted to continue in 
future five years through the above predicted analysis. This will result in a serious health problem in China. According to our forecast analysis, the predicted mortality of the curve estimation and ARIMA model were more accurate than the GM model and the joinpoint regression analysis.

To focus on strengthening the prevention and treatment of esophageal cancer. Morbidity and mortality of esophageal cancer showed a steady upward trend in recent years, but compared with urban areas, mortality of rural areas is rising faster and higher, and the mortality in males is higher than in females. This suggests that prevention needs to focus on rural populations and men. We found that the mortality for EC would steadily rise in future five years, therefore, the prevention and control measures of EC should be taken eagerly. In addition, early detection and treatment of EC should be implemented in China, especially in high-risk populations, and national clinical diagnosis and treatment standards should be promoted, to enhance the diagnosis and treatment of esophageal cancer.

People over the age of 45 should actively prevent esophageal cancer. We show that esophageal cancer mortality increases rapidly in people over the age of 45 , reach the highest in the 80 to 84 age group, is higher in males and in rural areas. Many studies have shown that esophageal cancer incidence and mortality were higher for men than females in different regions of China. Chen et al shows that in many parts of rural in China, esophageal cancer is a serious threat to the health of local residents (Zhao, 2008; Zhao, 2009; Zhao, 2010; Zhao, 2011). Esophageal cancer is the result of synergy of the environment, diet and lifestyle and genetic factors. We should actively promote healthy eating, healthy lifestyle, improve the living environment, especially for rural men over the age of 45, esophageal cancer prevention and early diagnosis and treatment are necessary, thereby preventing the occurrence of esophageal cancer and reducing the incidence of mortality

Eastern, Central and Northern China is the focus region to prevent and treat the esophageal cancer. Trend surface analysis is one of the most widely used global surfacefitting procedure, the coefficients of the polynomial function are found by the method of least squares, insuring that the sum of the squared deviations from the trend surface is minimum. Trend-surface analysis on the registries shows that the Chinese EC mortality is in a geographical distribution of concentric circle. In addition, the mortality of EC in China shows two tendencies, the first major trend increased from southern China to eastern China, the second trend increased from northeast to the highest levels in the central region. Different spatial patterns in mortality are most likely related to demographic, socio-economic, health behaviors, and relevant risk factors.

Esophageal cancer mortality in eastern China's northern Jiangsu, Anhui and Fujian, Linzhou Henan Province in Central China and North China, Ci County, Hebei, Shanxi, Shenyang City, and Shanxi was higher than in other regions. Geographical distribution of esophageal cancer mortality was characteristic of concentric circles, this strange phenomenon prompted that environmental factors, especially the ambient nitrogen pollution might be associated with the occurrence of esophageal N-nitroso compounds (Lin et al., 2002; Lin et al., 2003; Lin et al., 2009).

Worldwide, esophageal cancer morbidity and mortality after lung cancer, colorectal cancer, stomach cancer and liver cancer, ranked No. 5, causing serious harm to human health. The incidence and mortality of esophageal cancer of China has been at a high level, ranking fourth in cause of cancer-related deaths overall, serious social and economic burden, and seriously affecting the healthy development of the local society and economy. Therefore the prevention and treatment of esophageal cancer is imperative. High risk group should take more physical activities and change lifestyles, such as no smoking, limiting alcohol consumption, and eating more fruits and vegetables. In addition, more exercise lifestyle interventions focusing on increasing physical activity may decrease the global burden of EC (Jemal et al., 2011).

We declare that we have no financial and personal relationships with other people or organizations that can inappropriately influence our work, there is no professional or other personal interest of any nature or kind in any product, service and/or company that could be constructed as influencing the position presented in, or the review of, the manuscript entitled.

\section{Acknowledgements}

The study was supported by grants from Guangdong Provincial Key Laboratory of Infectious Diseases and Molecular Immunopathology, China.

\section{References}

China health statistics yearbook 2006. Beijing, China union medical university press.

China health statistics yearbook 2007. Beijing, China union medical university press.

China health statistics yearbook 2008. Beijing, China union medical university press.

China health statistics yearbook 2009. Beijing, China union medical university press.

China health statistics yearbook 2011. Beijing, China union medical university press.

China health statistics yearbook 2012. Beijing, China union medical university press.

China statistical yearbook of health and family planning 2013. Beijing, China union medical university press.

Chen Z (2008). The third national mortality retrospective sampling survey report. Beijing, China union medical university press.

Chen Z, Chen Q, Xia H, Lin J (2011). Green tea drinking habits and esophageal cancer in southern China: a case-control study. Asian Pac J Cancer Prev, 12, 229-33.

Falk GW (2009). Risk factors for esophageal cancer development. Surg Oncol Clin N Am, 18, 469-85.

Herszenyi L, Tulassay Z (2010). Epidemiology of gastrointestinal and liver tumors. Eur Rev Med Pharmacol Sci, 14, 249-58.

Imdadullah M. (Retrieved 11 June 2014). "Time Series Analysis." Basic Statistics and Data Analysis, from itfeature.com

Jemal A, Bray F, Center MM, et al (2011). Global cancer statistics. CA Cancer J Clin, 61, 69-90.

Hao J, Zhao P, Chen Wq (2012). Chinese cancer registry annual report 2011. Beijing, military medical science press. 
Wen-Rui Tang et al

Keshavarzi B, Moore F, Najmeddin A, Rahmani F (2012). The role of selenium and selected trace elements in the etiology of esophageal cancer in high risk Golestan province of Iran. Sci Total Environ, 433, 89-97.

Lin K, Shen W, Shen Z, Wu Y, Lu S (2002). Dietary exposure and urinary excretion of total $\mathrm{N}$-nitroso compounds, nitrosamino acids and volatile nitrosamine in inhabitants of high- and low-risk areas for esophageal cancer in southern China. Int J Cancer, 102, 207-11.

Lin K, Shen W, Shen Z, Cai S, Wu Y (2003). Estimation of the potential for nitrosation and its inhibition in subjects from high- and low-risk areas for esophageal cancer in southern China. Int J Cancer, 107, 891-5.

Lin K, Wu Y, Shen W (2009). Interaction of total N-nitroso compounds in environment and in vivo on risk of esophageal cancer in the coastal area, China. Environ Int, 35, 376-81.

Zhao P, Chen Wq (2008). Chinese cancer registry annual report 2004. Beijing, China union medical university press.

Zhao P, Chen Wq (2009). Chinese cancer registry annual report 2008-cancer incidence and mortality in Chinese cancer registration areas in 2005. Beijing, military medical science press.

Zhao P, Chen Wq (2010). Chinese cancer registry annual report 2009-cancer incidence and mortality in chinese cancer registration areas in 2006. Beijing, military medical science press.

Zhao P, Chen Wq (2011). Chinese cancer registry annual report 2010-cancer incidence and mortality in chinese cancer registration areas in 2007. Beijing, military medical science press.

Shi XJ, Au WW, Wu KS, Chen LX, Lin K (2014). Mortality characteristics and prediction of female breast cancer in China from 1991 to 2011. Asian Pac J Cancer Prev, 15, 2785-91. 